\title{
STEMI due to Severe Blunt Chest Trauma
}

\section{Joseph Shiber, MD, FACP, FACEP, FCCM ${ }^{1 *}$, David Skarupa, MD, FACS ${ }^{2}$ and Timothy Hester, DO}

\author{
${ }^{1}$ Professor of Emergency Medicine, Neurology and Surgery, UF College of Medicine - Jacksonville, USA \\ ${ }^{2}$ Associate Professor of Surgery and Trauma - Critical Care, UF College of Medicine - Jacksonville, USA \\ ${ }^{3}$ Assistant Professor of Surgery, UF College of Medicine - Jacksonville, USA
}

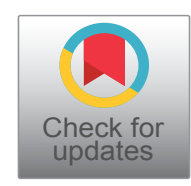

*Corresponding author: Joseph Shiber, MD, FACP, FACEP, FCCM, Professor of Emergency Medicine, Neurology and Surgery, UF College of Medicine - Jacksonville, USA

\section{Case Presentation}

A 50-year-old man was ejected during an ATV collision. On arrival to the trauma center, he was endotracheally intubated for hypoxemic respiratory distress and was noted to have palpable subcutaneous air and decreased breath sounds; thoracostomy tubes were placed bilaterally. New-onset atrial fibrillation with rapid ventricular response (heart rate $>160$ ) was found with ST-segment elevation most pronounced in Inferior leads (Figure 1) Chest radiograph confirmed severe right-sided pulmonary contusions (Figure 2) and CT showed multiple right and left-sided rib fractures and sternal fracture. He also sustained a subdural hematoma, subarachnoid hemorrhage, grade 1 hepatic injury, and left elbow fracture-dislocation. Repeat EKG after esmolol infusion showed return to sinus rhythm and confirmed ongoing ST-segment elevation. Bedside echocardiogram showed apical akinesis but no valvular abnormalities and left ventricular ejection fraction of $60 \%$. Emergent coronary catheterization revealed occlusions of distal LAD and RCA vessels; all other vessels were without disease. After a 3-week hospitalization requiring tracheostomy for prolonged mechanical ventilation he eventually had an excellent recovery.

\section{Discussion}

Myocardial infarction due to coronary artery injury is a rare complication of blunt chest trauma [1]. The Left Anterior Descending (LAD) artery is most commonly involved and the mechanism of injury include: 1 ) Intimal dissection with resultant thrombosis, 2) Vessel rupture,
3) Fissuring of a pre-existing atherosclerotic plaque, 4) Embolism (clot, marrow, air) to the coronary arteries, 5) Vessel spasm at the site of direct mechanical impact [2]. In our case, the presumed mechanism of the coronary artery thrombosis was direct impact causing localized thrombosis possibly from a combination of intimal injury and vasospasm. This type of myocardial infarction doesn't exactly fit into any of the five defined types: 1) Vessel plaque rupture - traditional coronary artery disease; 2) Supply/demand mismatch causing inadequate oxygen delivery to myocardium without coronary occlusion (such as from critical anemia, hypoxia, or global hypoperfusion as in severe circulatory shock); 3) Unexpected sudden cardiac death; 4) Iatrogenic MI due to PCl; and 5) latrogenic MI due to CABG [3]. Type 2 infarctions are the most common in multi-trauma patients due to hypotension, blood loss anemia, and hypoxemia which may have also contributed to the infarction in our patient. The diagnosis is still made by ECG, echocardiography, and serum cardiac enzymes but the presentation may be complicated by traumatic injuries $[1,2]$. The treatment continues to be revascularization either by percutaneous coronary intervention or coronary artery bypass grafting since there may be concern over significant bleeding risk with the use of systemic thrombolysis. Other Acute Coronary Syndromes may be managed with usual conservative medical therapies [4]. Aspirin, clopidogrel, and oral anticoagulants (such as warfarin) use for the treatment of myocardial infarction and post- $\mathrm{PCl}$ may be contraindicated in trauma patients with traumatic brain injuries or other injuries before definitive hemorrhage control is achieved (eg: grade 4 splenic laceration prior to angioembolization 


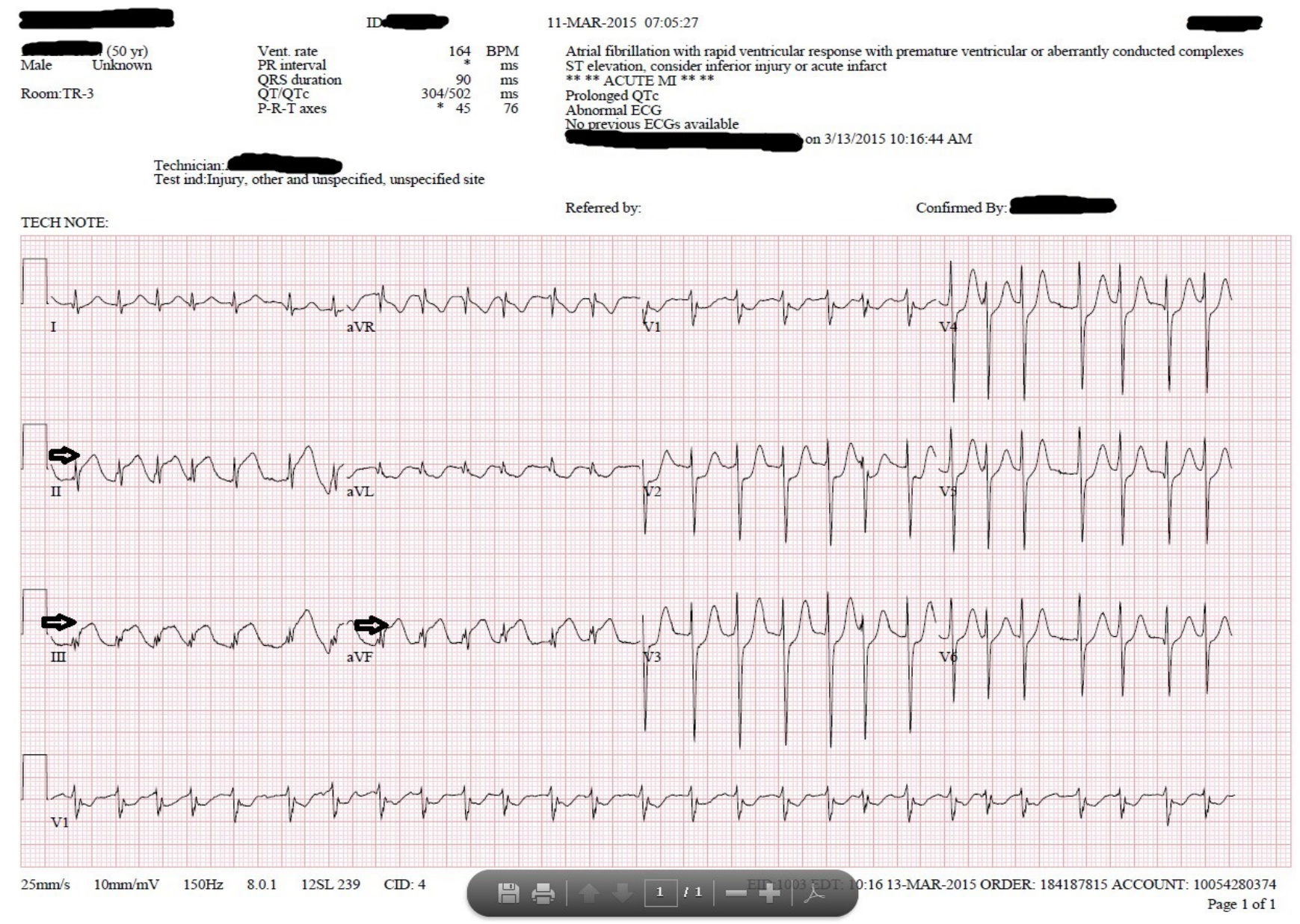

Figure 1: Initial EKG showing rapid atrial fibrillation and inferior ST elevation (arrows).

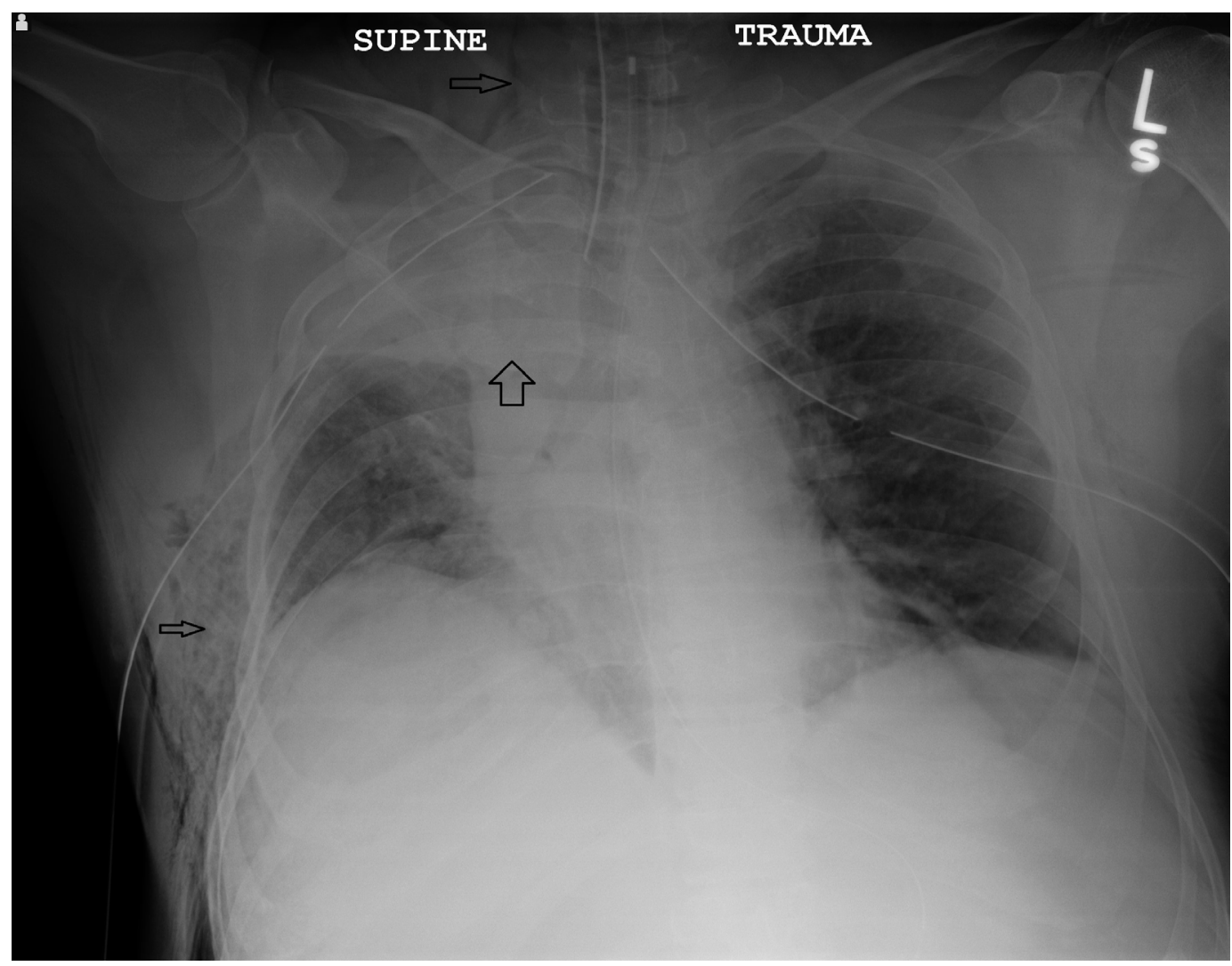

Figure 2: Anterior-posterior chest radiograph after bilateral thoracostomy tubes showing right upper lobe density (wide arrow) and right-sided subcutaneous air in chest wall and neck (narrow arrow). 
or splenectomy). It appears to be safe to restart these medications 7-14 days after traumatic brain injury in patients who have true ongoing indications [5].

\section{References}

1. Lolay GA, Abdel-Latif AK (2016) Trauma induced myocardial infarction. Int J Card 203: 19-21.

2. Colombo F, Zuffi A, Lupi A (2014) Left main dissection complicating blunt chest trauma: Case report and review of the literature. Cardiovasc Revasc Med 15: 354-356.
3. Thygesen K, Alpert JS, White HD, Joint ESC/ACCF/AHA/ WHF Task Force for the Redefinition of Myocardial Infarction (2007) Universal definition of myocardial infarction. Eur Heart J 28: 2525-2538.

4. Ginzburg E, Dygert J, Parra-Davila E, Lynn M, Almeida J, et al. (1998) Coronary artery stenting for occlusive dissection after blunt chest trauma. J Trauma 45: 157-161.

5. Puckett $\mathrm{Y}$, Zhang $\mathrm{K}$, Blasingame J, Lorenzana J, Parameswaran S, et al. (2018) Safest time to resume oral anticoagulation in patients with traumatic brain injury. Cureus 10: e2920. 